\title{
News, Tipps und Termine
}

\author{
Konrad Krug und Thomas Vogt
}

\section{Herausragend}

Karen Uhlenbeck ist die diesjährige Abelpreisträgerin. Dies hat die norwegische Akademie der Wissenschaften am 19. März bekanntgegeben. Die US-Amerikanerin ist damit die erste Frau, der diese Ehre zuteil wurde.

Ausgezeichnet wurde sie „für ihre Pionierleistungen bei geometrischen partiellen Differentialgleichun- gen, Eichtheorie und integrablen Systemen sowie für die grundlegenden Auswirkungen ihrer Arbeit auf Analysis, Geometrie und mathematische Physik."

In ihrer Arbeit beschäftigt sich die 76-jährige Mathematikerin mit Differentialgeometrie und partiellen Differentialgleichungen. Anwendung findet ihre Forschung unter Anderem in der Quantenfeldtheorie.

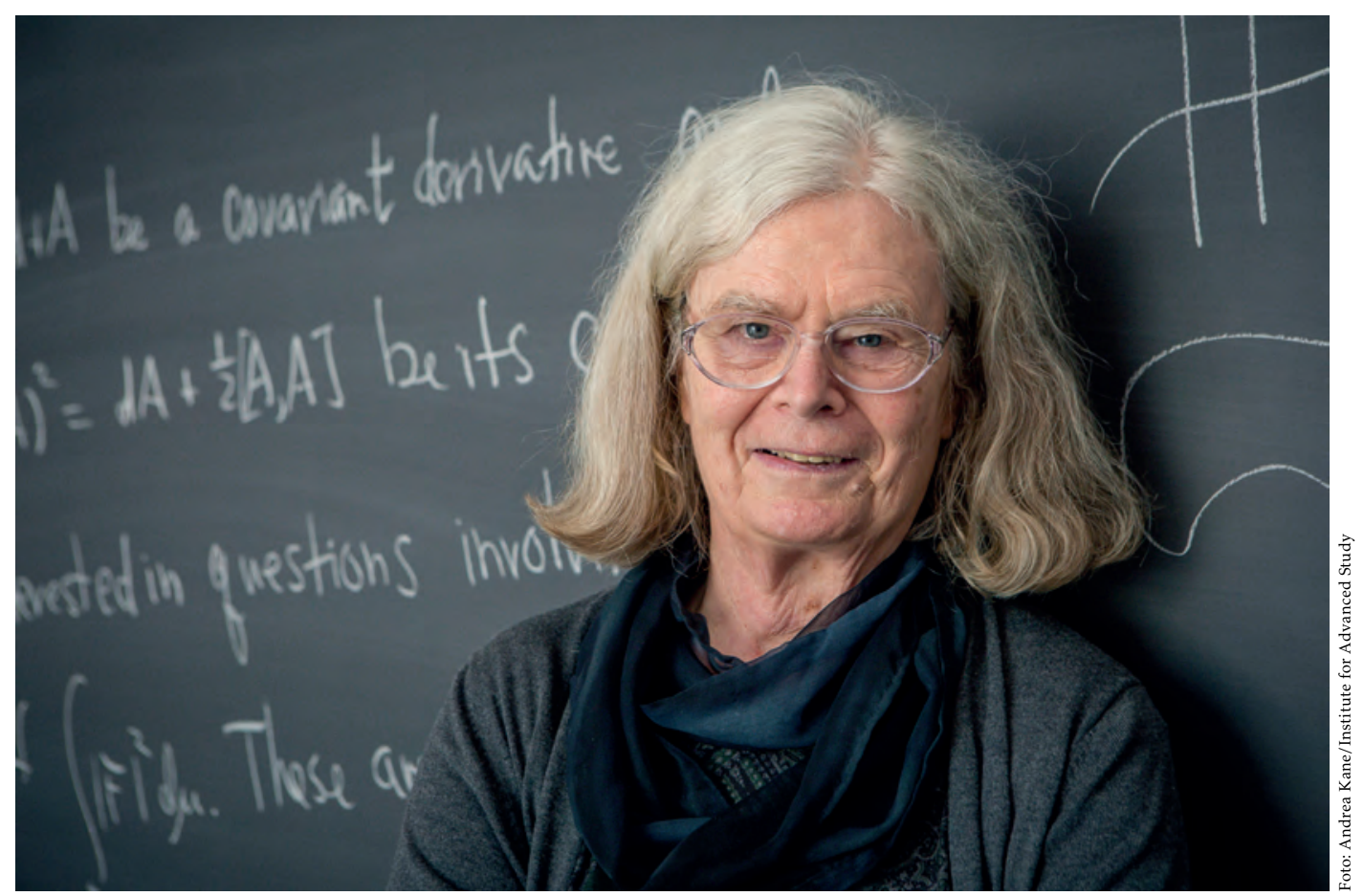

Karen Uhlenbeck, Abelpreisträgerin 2019

\section{Ausgezeichnet}

Je eine Mathematikerin und ein Mathematiker erhalten in diesem Jahr eine besondere Auszeichnung, wie die Alexander von Humboldt-Stiftung mit der folgenden Mitteilung bekannt gegeben hat.

Neun Spitzenwissenschaftler aus dem Ausland sind am 9. Mai in Berlin mit dem (mit bis zu 5 Millionen Euro) höchstdotierten Forschungspreis Deutschlands ausge- zeichnet worden, der Alexander von Humboldt-Professur. Der Präsident der Humboldt-Stiftung Hans-Christian Pape überreichte die Preise während einer Festveranstaltung mit der Bundesministerin für Bildung und Forschung Anja Karliczek an Margaret Crofoot, Malte Gather, Anke Hoeffler, Jens Meiler, Alexandre Obertelli, Stefanie Petermichl, Dietmar Schmucker, Henning Walczak und 


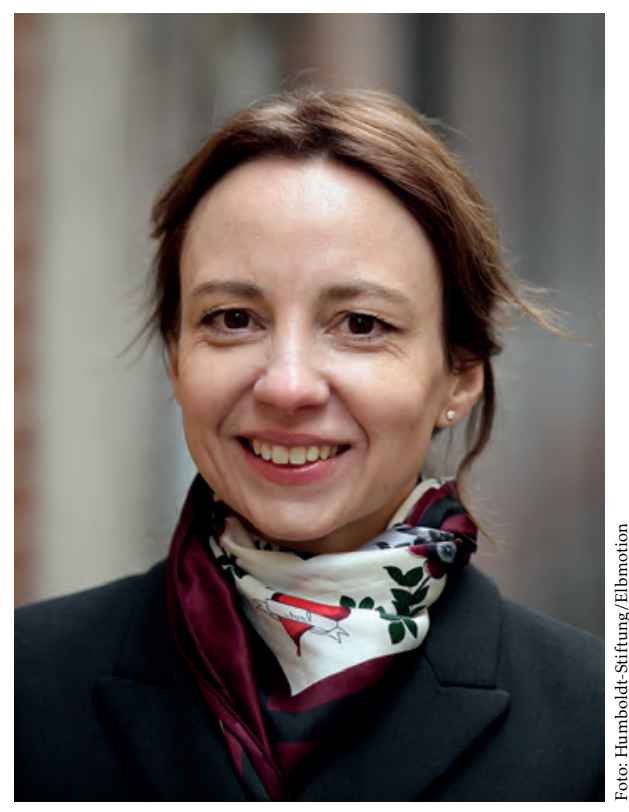

Stefanie Petermichel (Universität Toulouse)

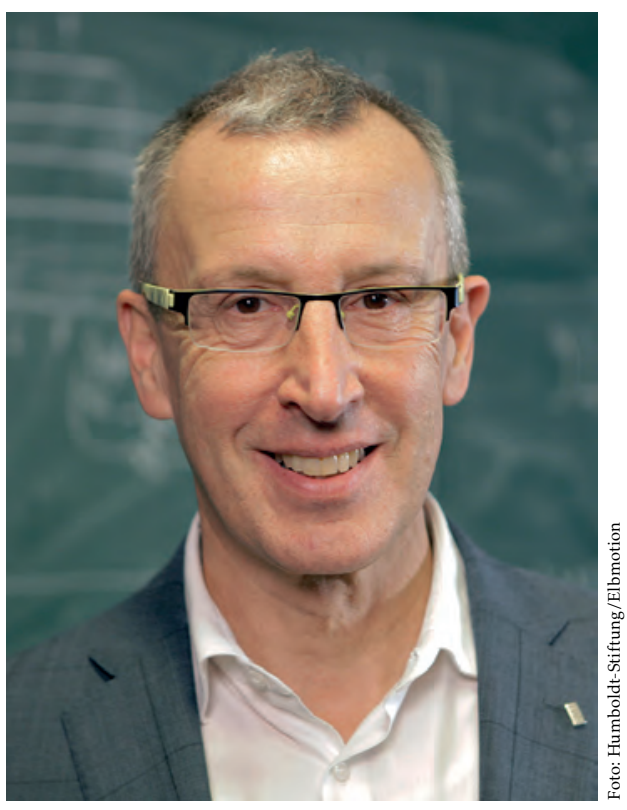

Enrique Zuazua (Autonome Universität Madrid)
Enrique Zuazua. Die Preisträgerinnen und Preisträger wurden von deutschen Hochschulen und Forschungseinrichtungen nominiert und werden nun in Bonn, Darmstadt, Erlangen-Nürnberg, Köln, Konstanz, Leipzig und Würzburg forschen.

Die Mathematikerin Stefanie Petermichl ist eine der führenden Forscherinnen auf dem Gebiet der harmonischen Analysis, einem Teilbereich der Mathematik mit breiten Anwendungsmöglichkeiten. So kann die harmonische Analysis helfen, medizinische Bildgebungsverfahren zu verbessern und wird in der Signalverarbeitung und den Materialwissenschaften genutzt. Stefanie Petermichl ist besonders für ihre Fähigkeit bekannt, die harmonische Analysis mit anderen mathematischen Fachrichtungen wie der Wahrscheinlichkeitstheorie zu verknüpfen. Mit außergewöhnlichen Ideen hat sie in den letzten Jahren das Forschungsfeld geprägt: So sind einige von ihr entwickelte Methoden inzwischen zu Standardwerkzeugen geworden, die entscheidende Fortschritte im Bereich der harmonischen Analysis erst ermöglicht haben.

Stefanie Petermichl wurde in Deutschland geboren. Sie ist Professorin am Mathematischen Institut der Université Toulouse III - Paul Sabatier in Frankreich, zuvor arbeitete sie bis 2009 an der Université de Bordeaux. In den USA war sie unter anderem von 2005 bis 2007 an der University of Texas in Austin und von 2002 bis 2005 an der Brown University in Providence tätig. Für ihre Forschungen wurde sie mehrfach ausgezeichnet: Sie erhielt einen ERC Consolidator Grant des Europäischen Forschungsrats, den Prix Ernest Déchelle der französischen Académie des sciences und als erste Frau den Prix Salem der Société Mathématique de France. Zudem wurde sie als Mitglied in das Institut Universitaire de France, einer staatlichen Einrichtung zur Förderung der Spitzenforschung und interdisziplinären Zusammenarbeit, gewählt.

Der Mathematiker Enrique Zuazua ist einer der international führenden Köpfe im Bereich der Angewandten Mathematik. Er beschäftigt sich unter anderem mit partiellen Differentialgleichungen, Kontrolltheorie und Numerik. Diese Felder der Mathematik zielen zum Beispiel darauf ab, Prozesse, die sich aus den Ingenieurwissenschaften ergeben, besser modellieren und simulieren zu können - in vielen besonders zukunftsträchtigen Bereichen wie Luftfahrt, Stromnetzen oder sozialem Verhalten. Auch für die angesichts des Klimawandels immer wichtigere Vorhersage von Naturphänomenen wie Flutwellen, sind die Qualität mathematischer Modelle sowie die zur Verfügung stehenden Bilder, Messwerte und Daten aus dem Forschungsfeld von Zuazua entscheidend.

Enrique Zuazua wurde in Spanien geboren und ist Leiter des Lehrstuhls für Computermathematik an der Universidad de Deusto in Bilbao sowie Professor für Angewandte Mathematik an der Universidad Autónoma in Madrid, Spanien. Zuvor war er, nach seinem Promotionsstudium in Spanien und Frankreich, an verschiedenen Forschungseinrichtungen in Spanien tätig. Zuazua ist außerdem Gründungsdirektor des 2008 eingerichteten Basque Centre for Applied Mathematics in Bilbao. Er erhielt zahlreiche Ehrungen und Preise, darunter zwei ERC Advanced Grants, den Humboldt-Forschungspreis sowie den nationalen Forschungspreis des spanischen Wissenschaftsministeriums ,Julio Rey Pastor" und die Ehrendoktorwürde der Université de Lorraine, Frankreich. 


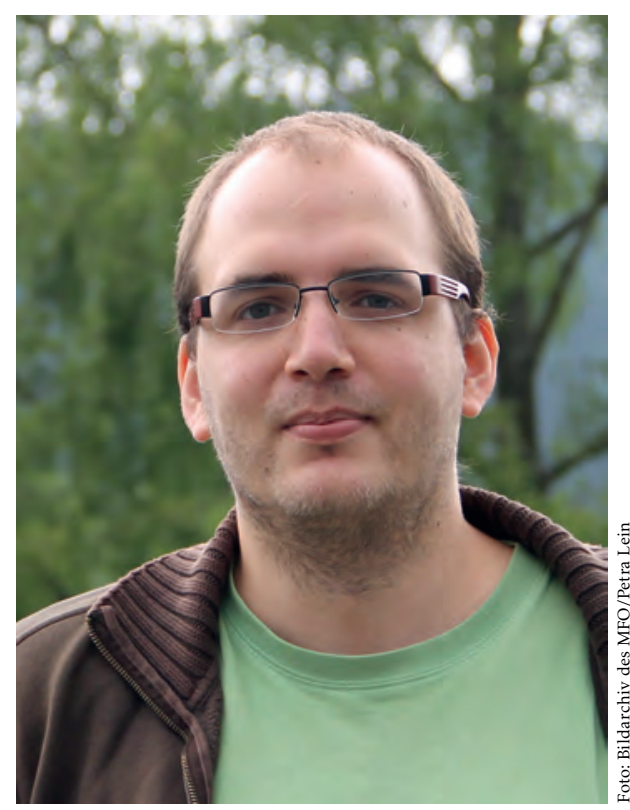

Karim Adiprasito (Hebrew University of Jerusalem)
Karim Adiprasito, Absolvent der FU Berlin und der Berlin Mathematical School (BMS), ist "for the development, with Eric Katz, of combinatorial Hodge theory leading to the resolution of the log-concavity conjecture of Rota" mit dem New-Horizons-Preis in Mathematik geehrt worden. Der von Facebook-Gründer Mark Zuckerberg, Anne Wojcicki und Juri Millner gestiftete Preis ist mit 100 ooo US-Dollar dotiert.

Adiprasito, der 2013 an der Freien Universität Berlin bei Günter M. Ziegler, DMV-Präsidiumsmitglied und ak- tueller FU-Präsident, mit summa cum laude promoviert wurde, ist damit einer der fünf Preisträger in der Kategorie Mathematik.

Für seine Dissertation mit dem Titel „Methods from differential geometry in polytope theory" hat Karim Adiprasito 2014 den Ernst-Reuter-Preis erhalten. 2015 wurde er mit dem European Prize in Combinatorics ausgezeichnet.

\section{Ausgezeichnet}

Am 4. April 2019 fand die Preisverleihung des ArsLegendi-Fakultätenpreises für Exzellente Hochschullehre in Mathematik und Naturwissenschaften statt: Ausgezeichnet wurden Robert Rockenfeller (Mathematik), Burkhard Priemer (Physik), Lena Daumann (Chemie), und Markus Piotrowski (Biologie). Der Ars-Legendi-Fakultätenpreis ist mit je 5000 Euro dotiert und ist eine der höchsten nationalen Auszeichnungen für universitäre Lehre im Bereich der Mathematik und Naturwissenschaften.

Vergeben wird der 2014 vom Stifterverband für die Deutsche Wissenschaft in Kooperation mit den großen Fachgesellschaften DMV, Deutsche Physikalische Gesellschaft (DPG), GDCh Gesellschaft Deutscher Chemiker (GDCh) und dem Verband Biologie, Biowissenschaften und Biomedizin in Deutschland (VBio) ins Leben gerufene Preis in den Kategorien Mathematik, Physik, Chemie und Biologie.

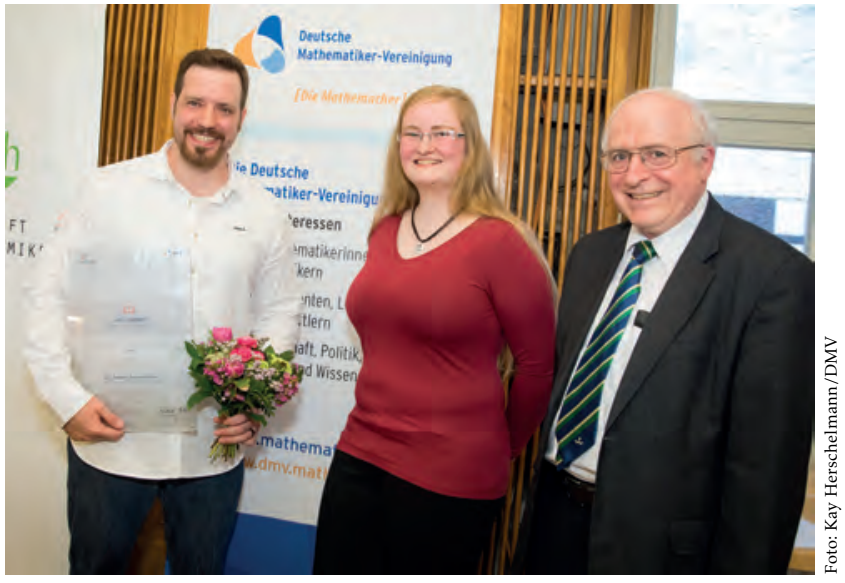

Robert Rockenfeller, Jessica Winter, Jurorin, und Friedrich Götze, Präsident der DMV (v. l.n. r.) 
Studierende der Mathematik sind aufgerufen, auf unseren Studierendenkonferenzen ihre im Jahr zuvor angefertigten Abschlussarbeiten (Bachelor, Diplom, Master oder Staatsexamen) vorzustellen. Dafür müssen die Studierenden und Absolventen ihre Arbeiten zuvor einreichen. Eine Jury bewertet dann die Arbeiten, wählt eine Reihe von ihnen zum Vortrag auf der Konferenz aus und verleiht verschiedene Preise. Die kommende Studierendenkonferenz ist Bestandteil der DMV-Jahrestagung 2019, die vom 23. bis 26. September in Karlsruhe stattfindet (s. S. 42).
Ansprechpartner für Interessierte sind Prof. Frank Loose, Uni Tübingen, und Dr. Stefan Kühnlein, KIT. Weitere Informationen auf dmv2019.math.kit.edu.

Die Studierendenkonferenz des Jahres 2020 findet am 16. und 17. Juli 2020 am MPI für Mathematik in den Naturwissenschaften in Leipzig statt.

Ansprechpartner sind Ilka Agricola, Uni Marburg, und Jörg Lehnert, Leipzig.

Ausgelobt

Die DMV lobt dieses Jahr wieder zwei Preise für die Präsentation von Mathematik aus: Den DMV-Medienpreis, dotiert mit 5000 Euro, gibt es für herausragende Leistungen bei der Vermittlung und Popularisierung von Mathematik. Er ist für Personen aus der Medienbranche, der Öffentlichkeitsarbeit oder der Wissenschaft gedacht, die Mathematik anschaulich und professionell an die brei- te Öffentlichkeit vermitteln. Beim zweiten Preis, dem DMV-Journalistenpreis, der mit 1000 Euro dotiert ist, wird ein Journalist oder eine Journalistin für einen besonders gelungenen Einzelbeitrag zur Mathematik in einem tagesaktuellen Medium ausgezeichnet.

Vorschläge und Bewerbungen sind bis 31. Juli 2019 möglich.

Ausgeschrieben

Die Deutsche Mathematiker-Vereinigung unterstützt Studierende der Mathematik und der Wirtschaftsmathematik, die eine Veranstaltung zur Mathematik planen und durchführen möchten. In der Förderlinie „DMVMathematikschulen“ fördert die DMV Veranstaltungen, die sich auf ein spezielles Gebiet in der Mathematik beziehen, auf bildungspolitische Fragen oder auch auf Berufsperspektiven nach einem Mathematikstudium. Möglich sind zwei Förderformate:

(1) Ein pauschaler Zuschuss in Höhe von 300 Euro zum Abdecken typischer Nebenkosten, etwa für Tee- und
Kaffeepausen, Posterdruck usw. Die Einreichung von Belegen entfällt.

(2) Ein Zuschuss von bis zu 1000 Euro für die Durchführung der Veranstaltung, wenn zum Beispiel auch Reise- und Aufenthaltskosten von Referenten anfallen. In dieser Förderlinie ist ein Budget vorzulegen, das die Höhe des beantragten Zuschusses begründet, zur Abrechnung sind Belege einzureichen.

Bewerbungen von bis zu dreiköpfigen Teams sind erbeten per E-Mail an Frau Prof. Ilka Agricola, E-Mail: dmv-mathematikschule@mathematik.de.

\section{Veröffentlicht}

Kürzlich hat der DAAD eine neue Ausschreibung für eine Hochschulkooperation mit den African Institute for Mathematical Science in Südafrika für den Zeitraum 2019-2023 veröffentlicht. Hintergrund: Seit 2012 unterstützt das BMBF durch den DAAD und die Alexandervon-Humboldt-Stiftung $(\mathrm{AvH})$ die Next Einstein Initiative. Die AvH realisiert dies über die Einrichtung von Forschungslehrstühlen an den African Institutes for Mathematical Science (AIMS-Zentren). Zur Internationalisierung dieser Forschungslehrstühle und um deutschen Hochschulen eine Einbindung in das weltweite AIMS-Netzwerk zu ermöglichen, fördert der DAAD Kooperationen deutscher Hochschulen und Forschungseinrichtungen mit den Lehrstühlen an den AIMSZentren.

Der DAAD fördert dabei aus Mitteln des BMBF Hochschulkooperationen von deutschen Hochschulen mit folgenden Standorten des African Institute for Mathematical Sciences (AIMS): Ghana, Kamerun, Senegal, Südafrika. Ausschreibung auf bit.ly/2JnRVEj. 
Am 6. Mai luden die drei großen deutschen mathematischen bzw. mathematikdidaktischen Fachgesellschaften DMV, GDM und MNU zum Pressegespräch in die Berliner Räume der Kultusministerkonferenz (KMK). Anlass waren die von der „Gemeinsamen Kommission der Fachgesellschaften " ausgearbeiteten Handlungsempfehlungen für einen leichteren Übergang von der Schule an die Hoch- schule. Der Maßnahmenkatalog sieht unter anderem eine deutschlandweite zentrale Abiturprüfung im Fach Mathematik und eine Erhöhung der Wochenstunden im Fach Mathematik auf mindestens vier Stunden, vor.

Das Pressegespräch stieß auch auf Resonanz bei den Medien: Unter anderem berichteten der Bayerische Rundfunk, Der Tagesspiegel und Zeit-Online darüber.

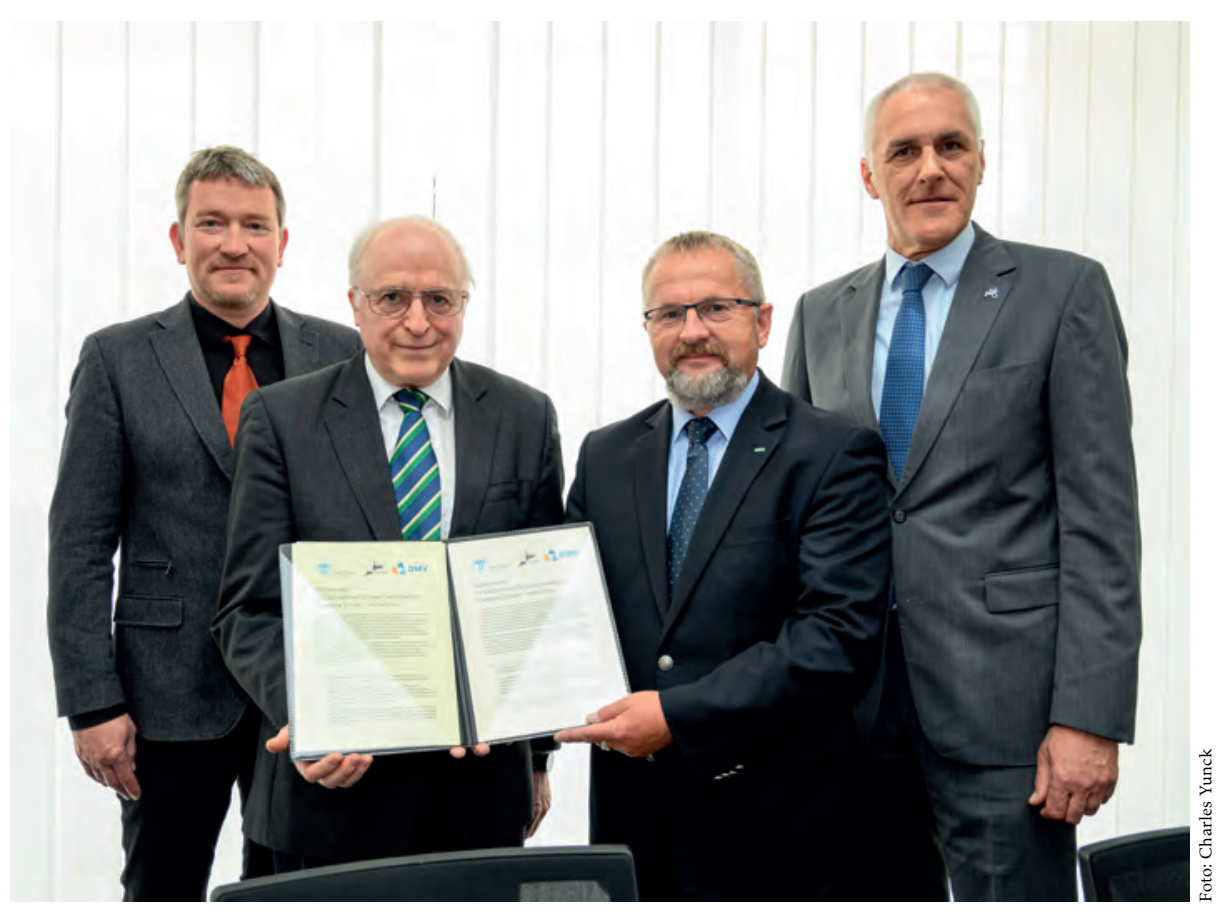

Andreas Eichler, 1. Vorsitzender der GDM), Friedrich Götze, Präsident der DMV, Udo Michalik, Generalsekretär der Kultusministerkonferenz, Gerald Heckmann, Vorsitzender des MNU (v. 1.n. r.)

Bindend

Die Fachgesellschaften GDCh (Chemie), DMV (Mathematik), DPG (Physik) und VBio (Biologie) bekennen sich zu den Leitlinien guter Wissenschaftskommunikation; dies wurde im Februar 2019 während eines gemeinsamen Treffens in Berlin beschlossen. Die Leitlinien sind ab sofort bindend für alle Kommunikatorinnen und Kommunikatoren dieser Fachgesellschaften. Erarbeitet wur- den sie von einem überinstitutionellen Arbeitskreis von Vertreter_innen aus Hochschulen, außeruniversitären Einrichtungen, Stiftungen und weiteren Institutionen basierend auf dem "Siggener Aufruf“. Hintergrund ist der „Krise des Wissenschaftsjournalismus“ bzw. „Krise der Wissenschafts-PR“ genannte Strukturwandel im Wissenschaftsjournalismus.
Eine Konferenz der besonderen Art findet vom 16. bis 20. Juli im Österreichischen Linz statt. Im Ars Electronica Center und an der Johannes-Keppler-Universität Linz sind Mathematiker_innen und Kunstschaffende eingeladen, um Brücken zu bauen zwischen den scheinbar so weit von einander entfernten Domänen. In Workshops und Gesprä- chen, bei Ausstellungen und bei Vorträgen kommen auf der „BRIDGES-Konferenz“ Akteure beider Disziplinen zusammen, um gemeinsam zu arbeiten, zu diskutieren und um gegenseitig nach Inspiration zu suchen.

Begleitet wird das Programm von Performances, Theater und Musik. 


\section{Termine}

$\triangleright$ 10.-22.7.2019, Bath, Großbritannien 6o. Internationale Mathematik Olympiade (IMO) www.imo2019.uk

$\triangleright$ 15.-19.7.2019, Valencia, Spanien International Congress on Industrial and Applied Mathematics (ICIAM) iciam2019.org/index.php

$\triangleright$ 22.-27.9.2019, Heidelberg $7^{\text {th Heidelberg Laureate Forum (HLF) }}$ www.heidelberg-laureate-forum.org $\triangleright$ 23.-26.9.2019, Karlsruhe DMV-Jahrestagung 2019 dmv2019.math.kit.edu

$\triangleright$ 23.-26.9.2019, Karlsruhe Studierendenkonferenz 2019 (im Rahmen der DMV-Jahrestagung) www.mathematik.de/dmv/jahrestagungen

$\triangleright$ 24.9. 2019: 17.00-18.0o Uhr, Karlsruhe DMV-Mitgliederversammlung dmv2019.math.kit.edu

Weitere News, Tipps, Termine auf mathematik.de sowie auf Facebook und Twitter.

Gedenken

Der Mathematiker Felix Klein wäre am 25. April 170 Jahre alt geworden. Er gilt als einer der bedeutendsten Mathematiker des ausgehenden 19. und frühen 20. Jahrhunderts. Mit seinem Erlanger Programm revolutionierte Klein die Geometrie, indem er sie als Invariantentheorie geometrischer Transformationen definierte und so einer modernen mathematischen Betrachtungsweise zugänglich machte. Auch als Wissenschaftsorganisator machte sich Klein einen Namen: Unter seiner Ägide entwickelte sich Göttingen zum Weltzentrum der Mathematik.

Felix Klein war dreimal Präsident der Deutschen Mathematiker-Vereinigung und blieb zeit seines Lebens DMV-Ehrenmitglied. 